\title{
Guillain-Barré Syndrome Variant with Facial Diplegia and Paresthesias Associated with IgM Anti-GalNAc-GD1a Antibodies
}

\author{
Ryuichiro Hayashi and Shigeki Yamaguchi
}

\begin{abstract}
We herein report the case of a 19-year-old woman with facial diplegia and paresthesias (FDP) preceded by flu-like symptoms. We diagnosed the patient with a regional variant of Guillain-Barré syndrome due to decreased tendon reflexes, albuminocytological dissociation in the cerebrospinal fluid and demyelinating features on nerve conduction studies. The patient also had IgM anti-GalNAc-GD1a antibodies, and treatment with glucocorticoids was effective for treating the facial diplegia, but not paresthesia. Therefore, facial palsy may have a different pathophysiology from paresthesia or other symptoms of FDP, which responds to glucocorticoid therapy.
\end{abstract}

Key words: Guillain-Barré syndrome, facial diplegia and paresthesia, IgM anti-GalNAc GD1a antiganglioside antibody, Epstein-Barr virus, glucocorticoid therapy

(Intern Med 54: 345-347, 2015)

(DOI: 10.2169/internalmedicine.54.2972)

\section{Introduction}

Facial diplegia and paresthesias (FDP) has been reported to be a regional variant of Guillain-Barré syndrome (GBS) (1). In this report, we describe a case of FDP in which IgM-GalNAc-GD1a antibodies were positive and glucocorticoid therapy was effective for treating the patient's facial diplegia.

\section{Case Report}

A 19-year-old woman was admitted to our hospital due to paresthesia of the extremities with muscle pain and right facial palsy. The patient had developed a headache and left retroauricular pain in the beginning of May. Although she sought medical attention and underwent magnetic resonance imaging (MRI) of the brain, no abnormalities were noted on MRI, and her pain gradually resolved. However, in the beginning of June, she suffered from mild diarrhea, fatigue and a sore throat, followed by the recurrence of retroauricular pain, which became bilateral, especially when she opened her mouth widely, associated with neck pain and paresthesia of the fingers and toes. She consulted another doctor and was prescribed analgesics and antibiotics for "pharyngitis." By the middle of June, the paresthesia had spread to the tongue, arms and legs. In addition, the patient's sense of taste had decreased and her retroauricular pain had worsened. She also subsequently noticed rightsided facial weakness and was unable to ascend the stairs due to thigh muscle pain. Fever, diplopia, hearing disturbances and skin eruptions were absent. Approximately 10 days after the appearance of paresthesia of the fingers and toes, she was referred to our hospital and admitted for a further examination.

The patient had no history of serious illnesses, operations or hospitalization. She was a university student and denied the use of illicit drugs, cigarettes and alcohol. She had been vaccinated against rubella and measles two years previously.

On a physical examination, the patient was alert and oriented and her speech was normal. Her blood pressure was $105 / 75 \mathrm{mmHg}$, with a pulse of 87 beats per minute and a temperature of $36.7^{\circ} \mathrm{C}$. No lymphadenopathy, swelling of the parotid glands or cutaneous eruptions were observed. How- 
ever, she exhibited incomplete right eye closure and was unable to move her forehead or right lip. Her capacity for left eye closure was also mildly weak and pinprick sensations were decreased on the right side of her face; however, other cranial nerves were intact. In addition, although she was unable to move her arms or legs fully due to myalgia, her muscle strength was considered to be intact. Meanwhile, deep tendon reflexes were normal in the upper extremities, but weak or absent in the lower extremities. The paresthesia affected the patient's palms and fingers of her hands, as well as her toes, ankles and soles of her feet. However, pinprick and joint position senses were normal in these areas. Vibration senses were slightly decreased in the extremities, and no ataxia affecting the limbs or trunk was noted.

Laboratory studies revealed mild elevation of liver enzymes; however, the blood count and serum levels of creatinine, electrolytes, C-reactive protein and anti-nuclear antibodies were within the normal limits. No atypical lymphocytes were detected, and an anti-HIV antibody test was negative. Meanwhile, a cerebrospinal fluid (CSF) study demonstrated a high protein concentration (140.9 mg/dL), with four white blood cells per cubic millimeter. Most cells in the CSF were lymphocytes, and a cytological examination showed a few of these lymphocytes to be atypical. The results of a urinalysis, electrocardiogram and chest X-rays were normal, as was an MRI of the brain without enhancement.

Serum immunoglobulin (Ig) G and IgM antibodies to glycolipids GM1, GM2, GM3, GD1a, GD1b, GD3, GT1b, GQ1b, galactocerebroside and GalNAc-GD1a were assessed; however, only IgM anti-GalNAc-GDla antibodies were detected. Although the viral titers for herpes simplex, varicella zoster, cytomegalovirus (CMV), measles, rubella, mumps, and Epstein-Barr virus (EBV) were determined using an electroimmunoassay, only EB viral capsid antigen (VCA) IgM was detected, with no significant increases in any viral IgG titers. Viral polymerase chain reaction (PCR) tests of the CSF were not performed. However, motor and sensory nerve conduction studies of the left upper and lower limbs showed a delayed tibial nerve conduction velocity $(38.3 \mathrm{~m} /$ s), whereas other nerve conduction velocities were almost normal and no conduction block was noted.

On the morning of the third day of admission, the patient's left facial palsy worsened. She was unable to completely close her eyes or mouth and needed to hold her lower lip while eating. The limb paresthesia persisted, and she felt pain when touching water during hand washing. Due to the patient's worsening bilateral facial weakness, treatment with intravenous methylprednisolone at a dose of $1,000 \mathrm{mg} /$ day (for three days, followed by prednisolone at a dose of $30 \mathrm{mg} /$ day, which was gradually tapered) and valaciclovir at a dose of 3,000 $\mathrm{mg}$ /day orally (for seven days) was administered. On the fourth day of admission, she was weakly able to close her eyes, eat without the need to hold her lower lip and partially taste salty foods. On the fifth day of admission, her capacity for right eye closure and right lip movement became almost normal. On the sixth day of hospitalization, the number of areas affected by paresthesia began to decrease. On the eighth day of hospitalization, her facial weakness and taste disturbances had almost completely resolved and the myalgia had disappeared. On the tenth day of hospitalization, she was discharged with persistent paresthesia of the fingers and toes. Two weeks after discharge, a follow-up examination was conducted at the same hospital, which showed continued mild limb paresthesia and retroauricular pain as well as weak deep tendon reflexes. At that point, the tibial motor conduction velocity had normalized; however, the distal compound muscle action potential exhibited a reduced amplitude $(1.79 \mathrm{mV})$ with polyphasic waves and a prolonged duration, thought to be indicative of temporal dispersion. In addition, the F-wave frequency was decreased at $20 \%$ (compared to $100 \%$ in the previous examination), whereas other motor and sensory conduction studies were unremarkable. Six months later, the patient's reflexes had recovered, the VCA IgM antibody titer was negative and the VCA IgG antibody titer was positive.

\section{Discussion}

Ropper reported "facial diplegia and paresthesias" as a regional variant of GBS (1) that includes features of facial diplegia, limb paresthesia, decreased deep tendon reflexes, albuminocytological dissociation in the CSF and demyelination on nerve conduction studies. Most patients with this rare variant of GBS present with limb numbness as the initial symptom, followed by facial palsy (2). Taste disturbances are common, although hearing, lacrimation and salivation are usually preserved (2). Our patient had these features, and her diagnosis was compatible with FDP.

Upper respiratory infection is a common preceding illness and anti-CMV antibodies are frequently positive in cases of FDP (2). It has also been reported that anti-EBV antibodies are more frequent in patients with FDP than in those with typical GBS (2). In the present case, despite the lack of specific symptoms (lymphadenopathy, splenomegaly and atypical lymphocytes in the serum), the antecedent infection was most likely caused by EBV based on the presence of EBV VCA IgM antibodies and seroconversion with the development of VCA IgG antibodies. EBV infection is sometimes complicated by the development of various neurological symptoms, especially facial diplegia $(3,4)$, and even paresthesia of all extremities (4). However, EBV-associated facial diplegia is usually accompanied by pleocytosis in the CSF (3). The present patient lacked CSF pleocytosis, although she was positive for serum IgM anti-GalNAc-GD1a antibodies. Therefore, we believe that the underlying pathophysiology in this case was an immune-mediated, postinfectious mechanism, not direct viral invasion.

The specific features of GBS patients with $\operatorname{IgM}$ antiGalNAc-GD1a antibodies have not been described $(5,6)$. However, Kaida et al. analyzed the clinical features of 29 GBS patients carrying IgM anti-GalNAc-GD1a antibodies, 
but not IgG class antibodies (5), and found that the subjects, particularly those with a history of an antecedent CMV infection, frequently presented with facial palsy and IgM antiGM2 antibodies. Therefore, the authors suggested that the presence of $\operatorname{IgM}$ antibodies against an epitope shared by GalNAc-GD1a and GM2 is associated with the development of facial palsy in GBS patients (5). Meanwhile, Susuki et al. investigated the relationship between FDP and antiganglioside antibodies (2). In their study of 22 FDP patients, seven had anti-ganglioside antibodies, most of which were IgM anti-GM2 antibodies, while only one patient had IgM anti-GalNAc-GD1a antibodies in addition to IgM anti-GM2 antibodies. Therefore, the contribution of IgM anti-GalNAcGD1a antibodies to the clinical manifestations of FDP remains unclear, although the detection of this antibody was helpful in diagnosing the present case as a GBS variant.

FDP patients often receive standard treatments for GBS (2). In recent reports of FDP, intravenous immunoglobulin (IVIG) or plasmapheresis has been frequently administered (7-9), whereas glucocorticoids have been reported to be ineffective (7). The present patient was able to walk independently on admission; therefore, we did not initiate therapy with IVIG or plasmapheresis. On the third hospital day, due to worsening of the patient's peripheral facial palsy and her persistent retroauricular pain, we started treatment with glucocorticoids and anti-viral agents. During the course of therapy, the facial diplegia, but not limb paresthesia, responded rapidly to the glucocorticoid therapy. Facial palsy has been reported to occur in some cases of variants of GBS, despite improvements in other neurological symptoms. For example, Chida et al. reported the development of facial palsy despite improvements in ataxia and ophthalmoplegia under immunomodulatory therapy in patients with Fisher syndrome (FS). The authors suggested that facial palsy is associated with unknown factors or involves a pathomechanism that differs from that of the cardinal signs of FS (10). The facial nerve runs through the bony canal and is vulnerable to compression injury, while nerve edema has many pathophysiological causes. Therefore, the rapid improvement in facial diplegia in response to glucocorticoids observed in the present case suggests that the anti-inflammatory and anti-edema effects of glucocorticoids helped to heal the patient's facial diplegia. A similar report described the case of an FDP patient with IgM anti-GalNAc-GD1a antibodies who responded to glucocorticoid therapy (11). Therefore, glucocorticoids may be useful for treating facial palsy in cases of FDP or other regional variants of GBS.

The authors state that they have no Conflict of Interest (COI).

\section{Acknowledgement}

We thank Prof. Susumu Kusunoki (Department of Neurology, Kinki University Medical School) for conducting the analysis of anti-glycolipid antibodies and Dr. Izumi Mori (Department of Neurology, Shonan Hospital) for her comments on the electrophysiological findings.

\section{References}

1. Ropper AH. Further regional variants of acute immune polyneuropathy. Bifacial weakness or sixth nerve paresis with paresthesias, lumbar polyradiculopathy, and ataxia with pharyngeal-cervicalbrachial weakness. Arch Neurol 51: 671-675, 1994.

2. Susuki K, Koga M, Hirata K, Isogai E, Yuki N. A Guillain-Barré syndrome variant with prominent facial diplegia. J Neurol 256: 1899-1905, 2009.

3. Diedler J, Rieger S, Koch A, Parthé-Peterhans S, Schwaninger M. Bilateral facial palsy: Epstein-Barr virus, not Lyme disease. Eur J Neurol 13: 1029-1030, 2006.

4. Matsushima K, Shinohara Y, Yamamoto M. A case of facial diplegia due to $\mathrm{EB}$ virus infection. Rinsho Shinkeigaku (Clin Neurol) 30: 1013-1016, 1990 (in Japanese, Abstract in English).

5. Kaida K, Kusunoki S, Kamakura K, Motoyoshi K, Kanazawa I. Guillain-Barré syndrome with $\operatorname{IgM}$ antibody to the ganglioside GalNAc-GD1a. J Neuroimmunol 113: 260-267, 2001.

6. Tatsumoto M, Koga M, Gilbert M, et al. Spectrum of neurological diseases associated with antibodies to minor gangliosides GM1b and GalNAc-GD1a. J Neuroimmunol 177: 201-208, 2006.

7. Lehmann HC, Macht S, Jander S, Hartung HP, Methner A. Guillain-Barré syndrome variant with prominent facial diplegia, limb paresthesia, and brisk reflexes. J Neurol 259: 370-371, 2012.

8. Barbi F, Ariatti A, Funakoshi K, Meacci M, Odaka M, Galassi G. Parvovirus B19 infection antedating Guillain-Barré syndrome variant with prominent facial diplegia. J Neurol 258: 1551-1552, 2011.

9. Yardimci N, Avci AY, Kayhan E, Benli S. Bilateral facial nerve enhancement demonstrated by magnetic resonance imaging in Guillain-Barré syndrome. Neurol Sci 30: 431-433, 2009.

10. Chida K, Takase S, Itoyama Y. Development of facial palsy during immunoadsorption plasmapheresis in Miller Fisher syndrome: a clinical report of two cases. J Neurol Neurosurg Psychiatry 64: 399-401, 1998.

11. Takiyama Y, Sato Y, Sawada M, Nishizawa M, Nakano I, Kusunoki S. An unusual case of facial diplegia. Muscle Nerve 22: 778-779, 1999.

(C) 2015 The Japanese Society of Internal Medicine http://www.naika.or.jp/imonline/index.html 